Pacific Journal of Mathematics

EXCURSIONS ABOVE HIGH LEVELS FOR STATIONARY 


\title{
EXCURSIONS ABOVE HIGH LEVELS FOR STATIONARY GAUSSIAN PROCESSES
}

\author{
SimeON M. Berman
}

\begin{abstract}
Let $X(t), t \geqq 0$, be a real valued stationary Gaussian process with mean 0 , variance 1 , covariance function $r(t)$, and continuous sample functions. For $u>0$ and $T>0$ let $L$ be the Lebesgue measure of the set $\{t: 0 \leqq t \leqq T, X(t)>u\}$, i.e., the time spent above $u$ in $[0, T]$. This paper proves: If $r$ is nonperiodic, and $r(t)=1-1 / 2 \gamma^{2} t^{2}+o\left(t^{2}\right), t \rightarrow 0$, for some $\gamma>0$, then the conditional distribution of $\gamma u L$, given $L>0$, converges for $u \rightarrow \infty$ to the distribution $1-\exp$ $\left(-x^{2} / 8\right)$.
\end{abstract}

This type of theorem - on the conditional limiting distribution of excursions above high levels - was first discovered by Palmer [7] and Rice [8]. Their work was clarified and generalized by Kac and Slepian [5]. The less general result of Volkonskii and Rozanov [10] was independently obtained about that time; it is included in the monograph of Cramér and Leadbetter [4]. The most recent generalization is that of Beljaev and Nosko [2]. One of the problems in the formulation of this theorem is that the conditional distribution must be correctly and usefully defined. While our definition of the conditional distribution is slightly different from that of the previous authors, our hypothesis is the most general because it requires only the finiteness of the second spectral moment.

The main idea of our proof is this: If the sample function spends positive time above a high level, then the duration of the excursion is very small; therefore, it is sufficient to consider the process only over a small interval. On such an interval the process is approximable by a linear combination of a sine and cosine function with independent Gaussian coefficients. The limit theorem is shown to hold for this particular kind of process; then, it is extended to the more general process.

1. Excursions for random trigonometric functions. Let $Y_{1}$ and $Y_{2}$ be independent random variables with a common standard Gaussian distribution. Put

$$
Y_{1}=R \cos \theta, \quad Y_{2}=R \sin \theta, R>0, \quad 0 \leqq \theta<2 \pi ;
$$

then, as is well known, the random variables $R$ and $\theta$ are independent. $R$ has the Rayleigh distribution 


$$
\begin{aligned}
\Psi(t) & =0, & & t \leqq 0 \\
& =1-\exp \left(-t^{2} / 2\right), & & t>0,
\end{aligned}
$$

and $\theta$ has the uniform distribution. We put:

$$
\psi(t)=\frac{d \Psi}{d t}, \quad t \neq 0 .
$$

The random function

$$
Y(t)=Y_{1} \cos t+Y_{2} \sin t, \quad t \geqq 0,
$$

is a stationary Gaussian process with mean 0 , unit variance and correlation function $\cos t$; it is also representable as

$$
Y(t)=R \cos (\theta+t),
$$$$
t \geqq 0 \text {. }
$$

In this section we shall prove that conditional limiting distribution of the excursion for $Y(t)$ exists and is equal to $\Psi$.

We need this elementary result from geometric probability.

Lemma 1.1. Let $A$ and $B$ be arcs of lengths $\alpha$ and $\beta$, respectively, on the circle of unit radius. Suppose $0<\alpha<\pi$ and $0<\beta<\pi / 2$. Let $A$ be fixed and $B$ uniformly distributed over the circle. (One endpoint is uniformly distributed and $\beta$ is fixed.) Let $L$ be the length of $A \cap B$; then $L$ has the distribution:

$$
\begin{aligned}
& P[L=0]=1-(\alpha+\beta) / 2 \pi \\
& \quad P[0<L<x]=x / \pi, \quad 0<x<\min (\alpha, \beta) \\
& P[L=\min (\alpha, \beta)]=|\alpha-\beta| / 2 \pi .
\end{aligned}
$$

Proof. It is evident from the rotation-invariance of the uniform distribution on the circle that the distribution of $L$ is the same as when $A$ is random and $B$ fixed; therefore, in proving the lemma we may, for definiteness, assume $\beta<\alpha$. Let $B$ be the arc whose endpoints have polar angles $\theta$ and $\theta+\beta$, respectively, where $\theta$ is uniformly distributed on $[0,2 \pi]$. We may suppose that $A$ has endpoints with the polar angles 0 and $\alpha$, respectively. $L$ is equal to 0 if and only if $\theta$ falls between $\alpha$ and $2 \pi-\beta . \quad L$ is equal to $\beta$ if and only if $\theta$ falls between 0 and $\alpha-\beta$. For $0<x<\beta, L$ assumes a value between 0 and $x$ if and only if $\theta$ falls in one or the other of the disjoint intervals : $(2 \pi-\beta, 2 \pi+x-\beta)$ and $(\alpha-x, \alpha)$. The statement of the lemma follows.

Lemma 1.2. Let $R$ and $\theta$ be independent random variables such that $R$ has the distribution $\Psi$ and $\theta$ has the uniform distribution on 
$[0,2 \pi]$. For fixed $u>0$ and $\tau, 0<\tau<\pi / 2$, let $L$ be the Lebesgue measure of

$$
\{s: 0 \leqq s \leqq \tau, R \cos (\theta+s)>u\} .
$$

Then $L$ has the distribution

$$
\begin{aligned}
& P[L=0]=1-\frac{1}{2 \pi} \int_{u}^{\infty}\left[\tau+2 \cos ^{-1}(u / t)\right] \psi(t) d t ; \\
& \begin{array}{l}
P[0<L<x]=(x / \pi) \exp \left[-u^{2} / 2 \cos ^{2}(x / 2)\right] \\
\quad+(1 / 2 \pi) \int_{u}^{u / \cos (x / 2)}\left[\tau+2 \cos ^{-1}(u / t)\right] \psi(t) d t, \quad 0<x<\tau ; \\
P[L=\tau]=(1 / 2 \pi) \int_{u / \cos (-/ 2)}^{\infty}\left[2 \cos ^{-1}(u / t)-\tau\right] \psi(t) d t .
\end{array}
\end{aligned}
$$

Proof. If $R>u$, then $L$ represents the length of intersection of an arc of (random) length $2 \cos ^{-1}(u / R)$ and a uniformly distributed arc of fixed length $\tau$. If $R \leqq u$, then $L=0$. We obtain the distribution of $L$ by conditioning with respect to $R$, noting the independence of $R$ and $\theta$, and applying Lemma 1.1 .

Write the probability as an integral of the conditional probability:

$$
P[L=0]=P[R \leqq u]+\int_{u}^{\infty} P[L=0 \mid R=t] \psi(t) d t .
$$

Put $\alpha=2 \cos ^{-1}(u / t), \beta=\tau$ and apply Lemma $1.1:$ the integrand above is equal to

$$
1-(1 / 2 \pi)\left[2 \cos ^{-1}(u / t)+\tau\right] .
$$

This establishes (1.3).

For $0<x<\tau$ we have

$$
\begin{aligned}
P[0<L<x] & =\int_{u}^{\infty} P[0<L<x \mid R=t] \psi(t) d t \\
& =\left(\int_{I_{1}}+\int_{I_{2}}\right) P[0<L<x \mid R=t] \psi(t) d t,
\end{aligned}
$$

where

$$
I_{1}=\{t: t \geqq u / \cos (\tau / 2)\} \text { and } I_{2}=\{t: u \leqq t \leqq u / \cos (\tau / 2)\} .
$$

If $t \in I_{1}$, then $x<\tau \leqq 2 \cos ^{-1}(u / t)$; thus, by Lemma 1.1 :

$$
P[0<L<x \mid R=t]=x / \pi, \quad t \in I_{1} .
$$

If $t \in I_{2}$, then $\min \left(\tau, 2 \cos ^{-1}(u / t)\right) \leqq 2 \cos ^{-1}(u / t)<x$; therefore, by Lemma 1.1: 


$$
\begin{aligned}
P[0<L<x \mid R & =t]=P\left[0<L \leqq \min \left(\tau, 2 \cos ^{-1}(u / R)\right) \mid R=t\right] \\
& =(1 / 2 \pi)\left[\tau+2 \cos ^{-1}(u / t)\right],
\end{aligned}
$$

Put these conditional probabilities into the integrals in (1.6); we obtain (1.4).

If $L=\tau$, then $2 \cos ^{-1}(u / R) \geqq \tau$; thus :

$$
P[L=\tau]=\int_{u / \cos (\tau / 2)}^{\infty} P[L=\tau \mid R=t] \psi(t) d t .
$$

Lemma 1.1 implies:

$$
P[L=\tau \mid R=t]=(1 / 2 \pi)\left[2 \cos ^{-1}(u / t)-\tau\right], \quad \text { for } u / \cos (\tau / 2) \leqq t .
$$

This confirms (1.5).

Now we find the limit of the conditional distribution

$$
P[L \leqq x / u \mid L>0]
$$

for fixed $x$ and for $u \rightarrow \infty$. This conditional distribution is equal to

$$
Q(x ; u)=\frac{P[0<L<x / u]}{P[L>0]}, \quad 0<x \leqq \tau u .
$$

We shall show that the moments of $Q$ converge to the corresponding moments of the distribution $\Psi(x / 2)$. Since $\Psi$ is evidently determined by its moment sequence, the well known convergence theorem [6, p. 185] implies that $Q(x ; u) \rightarrow \Psi(x / 2)$ for all $x$. Although it is easier to prove the latter convergence directly from the functional form of $Q$, it is necessary for our purposes to prove the stronger result on the convergence of the moments.

It follows from (1.7) that the $n^{\text {th }}$ moment of $Q$ is given by the formula :

$$
\int_{0}^{u \tau} x^{n} Q(d x ; u)=\frac{u^{n} \int_{0}^{\tau} x^{n} d P[0<L \leqq x]}{P[L>0]}, \quad n \geqq 1 .
$$

LEMMA 1.3 The moments of $Q$ converges to the corresponding moments of $\Psi(x / 2)$; this holds for all $\tau, 0<\tau<\pi / 2$.

Proof. By Lemma 1.2 and the definition of $\psi$ :

$$
P[L>\vartheta]=(\tau / 2 \pi) \exp \left(-u^{2} / 2\right)+(1 / \pi) \int_{u}^{\infty} \cos ^{-1}(u / t) \psi(t) d t .
$$

Put

$$
\Phi(u)=(1 \sqrt{2 \pi}) \int_{-\infty}^{u} \exp \left(-y^{2} / 2\right) d y ;
$$


then :

$$
(1 / \pi) \int_{u}^{\infty} \cos ^{-1}(u / t) \psi(t) d t=1-\Phi(u) .
$$

This is verified by noting: The members are equal for $u=0$. The derivative of the left hand member is

$$
-(1 / \pi) \int_{u}^{\infty}\left(t^{2}-u^{2}\right)^{-1 / 2} \psi(t) d t
$$

which, by the change of variable $y=t^{2}-u^{2}$, is seen to be equal to the derivative of the right hand member, for all $u$. The relation (1.9) is equivalent to

$$
P[L>0]=(\tau / 2 \pi) \exp \left(-u^{2} / 2\right)+1-\Phi(u) ;
$$

thus, from the well known relation

$$
1-\Phi(u) \sim(2 \pi)^{-1 / 2} u^{-1} \exp \left(-u^{2} / 2\right)
$$

we obtain :

$$
P[L>0]=(\tau / 2 \pi) \exp \left(-u^{2} / 2\right)\left[1+0\left(u^{-1}\right)\right], \quad u \rightarrow \infty .
$$

Since the distribution of $L$ has a jump at $x=\tau$, the moment (1.8) is equal to the sum of

$$
u^{n} \int_{0}^{\tau-} x^{n}(d / d x) P[0<L \leqq x] / P[L>0]
$$

and

$$
u^{n} \tau^{n} P[L=\tau] / P[L>0] .
$$

Differentiate the right hand member of (1.4):

$$
\begin{aligned}
\frac{d}{d x} P[0<L<x] & =\frac{\tau u^{2} \sin (x / 2) \exp \left[-u^{2} / 2 \cos ^{2}(x / 2)\right]}{4 \pi \cos ^{3}(x / 2)} \\
& +(1 / \pi) \exp \left[-u^{2} / 2 \cos ^{2}(x / 2)\right]\left\{1-\frac{x u^{2} \sin (x / 2)}{4 \cos ^{3}(x / 2)}\right\} .
\end{aligned}
$$

Substitute this in (1.12) and apply (1.11): the expression (1.12) is asymptotic to the sum of the two integrals

$$
\int_{0}^{\tau} \frac{(u x)^{n} u \sin (x / 2)}{2 \cos ^{3}(x / 2)} \exp \left[-\frac{u^{2}}{2}\left(\frac{1}{\cos ^{2}(x / 2)}-1\right)\right] u d x
$$

and

(1.15) $\frac{2}{\tau} \int_{0}^{\tau}(u x)^{n}\left\{1-\frac{x u^{2} \sin (x / 2)}{4 \cos ^{3}(x / 2)}\right\} \exp \left[-\frac{u^{2}}{2}\left(\frac{1}{\cos ^{2}(\tau / 2)}-1\right)\right] d x$. 
Change the variable of integration: $y=u x$. From the relation

$$
\cos ^{-2} t-1=t^{2}\left(1+0\left(t^{2}\right)\right), \quad t \rightarrow 0,
$$

we find that the integral (1.14) converges to the $n^{\text {th }}$ moment of $\Psi(x / 2)$, and that (1.15) converges to 0 . The expression (1.13) also converges to 0 ; indeed, by (1.5) and (1.11), it is equal to

$$
\exp \left(u^{2} / 2\right)(u \tau)^{n} \int_{u / \cos (\tau / 2)}^{\infty}\left[2 \cos ^{-1}(u / t)-\tau\right] \psi(t) d t\left(1+0\left(u^{-1}\right)\right),
$$

which is less than

$$
\text { constant } \cdot u^{n} \exp \left(-\frac{u^{2}}{2}\left[\frac{1}{\cos ^{2}(\tau / 2)}-1\right]\right),
$$

which tends to 0 as $u \rightarrow \infty$.

2. An inequality for the moments. Let $X(t)$ and $Y(t), t \in I$, where $I$ is some index set, be two Gaussian processes with means identically equal to 0 and with a common positive variance function. A well know result of Slepian [9] is: If

$$
E X(s) X(t) \leqq E Y(s) Y(t), \quad \text { for all } s, t \in I,
$$

then, for all $u$,

$$
P[\max (X(t): t \in I) \leqq u] \leqq P[\max (Y(t): t \in I) \leqq u] .
$$

(It is implicitly assumed that the maximum is a well defined random variable.) By applying (2.2) to the processes $-X(t)$ and $-Y(t)$, we also obtain: for all $u$,

$$
P[\min (X(t): t \in I) \geqq u] \leqq P[\min (Y(t): t \in I) \geqq u] .
$$

We remark that (2.2) and (2.3) are valid also when the inequalities on $u$ are replaced by strict inequalities.

LEMma 2.1. Let $X(t)$ and $Y(t), 0 \leqq t \leqq \tau$, be two Gaussian processes with continuous sample functions and satisfying the conditions above. Let $L_{1}$ and $L_{2}$ be the times spent above level $u$ by $X$ and $Y$, respectively; then

$$
E\left(L_{1}^{n} \mid L_{1}>0\right) \leqq E\left(L_{2}^{n} \mid L_{2}>0\right) .
$$

Proof. $L_{1}$ is the integral over $[s: 0 \leqq s \leqq \tau]$ of the indicator of the event $X(s)>u$; thus, by Fubini's theorem:

$$
E L_{1}^{n}=\int_{0}^{\tau} \cdots \int_{0}^{\tau} P\left[X\left(t_{j}\right)>u, j=1, \cdots, n\right] d t_{1}, \cdots, d t_{n} .
$$


By (2.3), with " $>$ ", in place of " $\geqq$ ", the integral is at most equal to the one corresponding to the process $Y$; therefore :

$$
E L_{1}^{n} \leqq E L_{2}^{n} \text {. }
$$

The events $\left\{L_{i}>0\right\}, i=1,2$ are equivalent to the events $\{\max (X(t): 0 \leqq t \leqq \tau)>u\}$ and $\{\max (Y(t): 0 \leqq t \leqq \tau)>u\}$, respectively; thus, by (2.2):

$$
P\left[L_{1}>0\right] \geqq P\left[L_{2}>0\right] .
$$

Since $L_{i}$ is nonnegative, we have:

$$
E\left(L_{i}^{n} \mid L_{i}>0\right)=E L_{i}^{n} / P\left[L_{i}>0\right], \quad i=1,2 ;
$$

thus, (2.4) follows from (2.5) and (2.6).

3. An asymptotic formula for the distribution of the maximum of a general process. Let $X(t), t \geqq 0$, be a real valued stationary Gaussian process with mean 0 , variance 1 and correlation function $r(t)$ which is continuous and has a finite second derivative at the origin. It is well known that $-r^{\prime \prime}(0)$ is equal to the second moment of the spectral distribution; we put

$$
\gamma=\sqrt{-r^{\prime \prime}(0)}
$$

so that

$$
1-r(t) \sim \gamma^{2} t^{2} / 2
$$

We assume :

$$
r(t)=1 \quad \text { if and only if } t=0 \text {; }
$$

finally, we suppose that $X$ has continuous sample functions.

Our object is to prove :

THEOREM 3.1. For any $T>0$ :

$$
P[\max (X(t): 0 \leqq t \leqq T)>u] \sim(T \gamma / 2 \pi) \exp \left(-u^{2} / 2\right),
$$
for $u \rightarrow \infty$.

The proof is long and will be completed after four lemmas. Note that the expression on the right hand side of (3.3) is identical with the well known Rice formula for the expected number of up-crossings of the level $u$ (see, for example, [4], p. 194). We have already established (3.3) for the particular process (1.1) and for $T<\pi / 2$ : the correlation function $\cos t$ satisfies (3.1) with $\gamma=1$, and (1.11) implies 


$$
P[\max (Y(t): 0 \leqq t \leqq T)>u] \sim(T / 2 \pi) \exp \left(-u^{2} / 2\right) .
$$

We shall extend (3.4) to (3.3)

Lemma 3.1. For every $\varepsilon, 0<\varepsilon<1$, there exists $\tau>0$ such that

$$
\begin{aligned}
& P[\max (Y(t): 0 \leqq t \leqq T \gamma(1-\varepsilon))>u] \leqq P[\max (X(t): \\
& 0 \leqq t \leqq T)>u] \leqq P[\max (Y(t): 0 \leqq t \leqq T \gamma(1+\varepsilon))>u]
\end{aligned}
$$

for all $u$ and all $T, 0 \leqq T \leqq \tau$.

Proof. The condition (3.1) implies:

$$
\lim _{t \rightarrow 0} \frac{1-r(t)}{1-\cos [t \gamma(1+\varepsilon)]}=(1+\varepsilon)^{-2}
$$

therefore, for every $\varepsilon, 0<\varepsilon<1$, there exists $\tau>0$ such that

$$
\cos [t \gamma(1+\varepsilon)] \leqq r(t) \leqq \cos [t \gamma(1-\varepsilon)], \quad|t|<\tau .
$$

The maximum of $Y(t), 0 \leqq t \leqq T \gamma(1 \pm \varepsilon)$, is equivalent to the maximum of the process $Y(t \gamma(1 \pm \varepsilon)), 0 \leqq t \leqq T$. The latter process has the covariance function $\cos (t \gamma(1 \pm \varepsilon))$. If $T \leqq \tau$, then, by (3.6), the covariance function of $X(t)$ dominates that of $Y(t \gamma(1+\varepsilon))$ and is dominated by that of $Y(t \gamma(1-\varepsilon))$ for $0 \leqq t \leqq T$. This and the inequality (2.2) imply (3.5).

Next we show that the estimate of the left hand side of (3.3) is changed by only a small amount if small pieces are removed from the domain of $X$ :

LEMMA 3.2. Let $m$ be a positive integer. Then there exist numbers $K>0$ and $\sigma>0$ such that:

If $J_{1}, \cdots, J_{m}$ are closed subintervals of $[0, T]$ each of length $h<\sigma$, and $J_{1} \cup, \cdots, \cup J_{m}$, then :

$$
\lim _{u \rightarrow \infty} \sup \frac{P[\max (X(t): t \in J)>u]}{P[\max (X(t): 0 \leqq t \leqq T)>u]} \leqq K h .
$$

Proof. Let $\varepsilon, 0<\varepsilon<1$, be arbitrary and fixed, and let $\sigma$ be the number $\tau$ in Lemma 3.1. By Boole's inequality and stationarity we have:

$$
P[\max (X(t): t \in J)>u] \leqq m P[\max (X(t): 0 \leqq t \leqq h)>u] .
$$

By the second inequality in (3.5) we find: 


$$
\begin{aligned}
& P[\max (X(t): 0 \leqq t \leqq h)>u] \leqq P[\max (Y(t): \\
&0 \leqq t \leqq h \gamma(1+\varepsilon))>u]
\end{aligned}
$$

Since $\tau$ may be taken to be arbitrarily small, we suppose that

$$
\sigma \gamma(1+\varepsilon)<\pi / 2 \quad \text { and } \quad \sigma<T .
$$

Equation (1.10) implies :

$$
\begin{aligned}
& P[\max (Y(t): 0 \leqq t \leqq h \gamma(1+\varepsilon))>u] \\
& \quad=(h \gamma / 2 \pi)(1+\varepsilon) \exp \left[-u^{2} / 2\right]+1-\Phi(u), u>0 .
\end{aligned}
$$

From (3.5) and (1.10) we obtain :

$$
\begin{array}{r}
P[\max (X(t): 0 \leqq t \leqq T)>u] \geqq P[\max (X(t): 0 \leqq t \leqq \sigma)>u] \\
\geqq(\sigma \gamma / 2 \pi)(1-\varepsilon) \exp \left(-u^{2} / 2\right)+1-\Phi(u) .
\end{array}
$$

As a consequence of (3.8), (3.9), (3.10) and (3.11), the ratio in (3.7) is at most equal to

$$
\frac{m h \gamma(1+\varepsilon) \exp \left(-u^{2} / 2\right) / 2 \pi+1-\Phi(u)}{\sigma \gamma(1-\varepsilon) \exp \left(-u^{2} / 2\right) / 2 \pi+1-\Phi(u)}, 0<h<\sigma, u>0 .
$$

The limit of this ratio for $u \rightarrow \infty$ is $K h$, where $K=m(1+\varepsilon) / \sigma(1-\varepsilon)$.

Let $I_{1}, \cdots, I_{m}$ be disjoint closed subintervals of $[0, T]$, and $I=I_{1} \cup \cdots \cup I_{m}$. We shall show that the maximum of $X$ over $I$ is asymptotically equivalent to the maximum over a sufficiently dense subset of $I$.

LEMmA 3.3. Let $g=g(u)$ be a positive increasing function of $u$ such that $g(u) \rightarrow \infty$ for $u \rightarrow \infty$. For each $u$ let $G_{k}=G_{k}(u)$ be the finite set of numbers which are of the form

$$
j T / u g(u), \quad \text { for some integer } j,
$$

and which belong to $I_{k}, k=1, \cdots, m$; put $G=G_{1} \cup \cdots \cup G_{m}$. Then :

$$
\lim _{u \rightarrow \infty} \frac{P[\max (X(t): t \in G)>u]}{P[\max (X(t): t \in I)>u]}=1 \text {. }
$$

Proof. The event in the numerator implies that in the denominator, so that the fraction is at most equal to 1 . It also follows that the difference between the probabilities in the denominator and numerator, respectively, is equal to the probability of the event

$$
A=\{X(t) \leqq u \text { for all } t \in G \text {, but } X(t)>u \text { for some } t \in I\} ;
$$

consequently, it suffices to prove: 


$$
\lim _{u \rightarrow \infty} \frac{P(A)}{P[\max (X(t): t \in I)>u]}=0 .
$$

The denominator in (3.12) does not increase when $I$ is replaced by an interval whose length is smaller than that of any of $I_{1}, \cdots, I_{m}$; therefore, by (3.11), the denominator is at least of the order exp $\left(-u^{2} / 2\right)$ for $u \rightarrow \infty$; hence, (3.12) is implied by

$$
\lim _{u \rightarrow \infty} \exp \left(u^{2} / 2\right) P(A)=0 \text {. }
$$

Let $N$ be the number of upcrossings of $u$ by $X(t), 0 \leqq t \leqq T$, and $N^{\prime}$ the number of upcrossings by the sampled sequence $X(j T / u g(u)), 0 \leqq j \leqq[u g(u)]$, that is, $N^{\prime}$ is the number of events

$$
\{X((j-1) T / u g(u)) \leqq u<X(j T / u g(u))\}, \quad 1 \leqq j \leqq[u g(u)],
$$

that occur. It is clear that $N^{\prime} \leqq N$. In order to avoid incidental complications, we shall suppose (without loss of generality) that the function $g$ has the property that endpoints of the intervals $I_{k}$ are of the form $j T / u g(u)$ for integral $j$. It follows that $A$ implies that $N-N^{\prime} \geqq 1$; thus, by Chebyshev's inequality :

$$
P(A) \leqq P\left[N-N^{\prime} \geqq 1\right] \leqq E N-E N^{\prime} .
$$

$E N$ is given by the right hand member of (3.3) (cf. remarks following (3.3)). By the above definition of $N^{\prime}$ and by stationarity we have :

$$
E N^{\prime}=[u g(u)] P[X(0) \leqq u<X(T / u g(u))]
$$

Writing

$$
P[X(0) \leqq u<X(s)]=P[X(s)>u]-P[X(0)>u, X(s)>u],
$$

and adapting the formula in $[4, \mathrm{p} .27]$, we find :

$$
P[X(0) \leqq u<X(T / u g(u))]=(2 \pi)^{-1} \int_{r(T / u g(u))}^{1} \exp \left[-u^{2} / 1+y\right]\left(1-y^{2}\right)^{-1 / 2} d y ;
$$

the latter is equal to

$$
(2 \pi)^{-1} \exp \left(-u^{2} / 2\right) \int_{r(T / u g(u))}^{1} \exp \left[-u^{2}(1-y) / 2(1+y)\right]\left(1-y^{2}\right)^{-1 / 2} d y .
$$

The integral in (3.16) is asymptotic to $T \gamma / u g(u)$ for $u \rightarrow \infty$; this can be verified by changing the variable of integration from $y$ to $1-y$ and applying the condition (3.1). From this and from (3.15), it follows that 
the latter and (3.14) imply (3.13). The proof is complete.

For each $u>0$ the maximum of $X$ on the finite set $G$ (cf. Lemma 3.3) is representable as the maximum of the $m$ sub-maxima $\max \left(X(t): t \in G_{k}\right), k=1, \cdots, m$. Now we shall prove that in estimating $P[\max (X(t): t \in G)>u]$ for $u \rightarrow \infty$ we may suppose that the sub-maxima are mutually independent random variables.

LEMMA 3.4. If, for some $p>0, g$ satisfies

$$
\lim _{u \rightarrow \infty}\left[g(u) / u^{P}\right]=0,
$$

then

$$
\lim _{u \rightarrow \infty} \frac{1-\prod_{k=1}^{m} P\left[\max \left(X(t): t \in G_{k}\right)<u\right]}{P[\max (X(t): t \in G)>u]}=1
$$

Proof. The numerator and denominator in (3.17) are equal if the $m$ sub-processes $\left\{X(t): t \in G_{k}\right\}, k=1, \cdots, m$, are mutually independent. A necessary and sufficient condition for this is

$$
r(s-t)=0, \text { for } s \in G_{i}, t \in G_{j}, i \neq j, i, j=1, \cdots, m .
$$

The point of this proof is that for a process with an arbitrary correlation the probability $P[\max (X(t): t \in G)>u]$ is asymptotically unchanged if the correlation is altered to satisfy the above relation.

By Lemma 3.3, the set $G$ in the denominator may be replaced by $I$; furthermore, by the remark preceding (3.13), the corresponding probability for $I$ is at least of the order $\exp \left(-u^{2} / 2\right)$; therefore, (3.17) is implied by the convergence to 0 of

$$
\begin{gathered}
\exp \left(u^{2} / 2\right) \cdot(\text { absolute difference between the numerator and } \\
\text { denominator in (3.17)). }
\end{gathered}
$$

We estimate the absolute difference by the method in [3]: Let $\left(X_{1}, \ldots, X_{n}\right)$ and $\left(Y_{1}, \ldots, Y_{n}\right)$ be two sets of Gaussian random variables with 0 means, unit variances, and covariance matrices $\left(r_{i j}\right)$ and $\left(s_{i j}\right)$, respectively; put $w_{i j}=\max \left(\left|r_{i j}\right|,\left|s_{i j}\right|\right)$; then from [3]:

$$
\begin{aligned}
\mid P\left[\max X_{i}\right. & >u]-P\left[\max Y_{i}>u\right] \mid \\
& \leqq(1 / 2 \pi) \sum_{i, \jmath=1}^{m}\left|r_{i j}-s_{i \jmath}\right|\left(1-w_{\imath j}^{2}\right)^{-1 / 2} \exp \left[-u^{2} / 1+w_{i j}\right] .
\end{aligned}
$$

Put $\left.r_{i j}=r(i-j) T / u g(u)\right)$, and define

$$
\begin{aligned}
s_{i j} & =r_{i j} \text { if } i T / u g(u) \text { and } j T / u g(u) \text { belong to a common } G_{k}, \\
& =0, \text { otherwise, } i, j=0,1, \cdots[u g(u)], k=1, \cdots, m .
\end{aligned}
$$

Since $I_{1}, \cdots, I_{m}$ are closed and disjoint, they are necessarily separated 
by nonempty open subintervals; therefore, there exists $\delta, 0<\delta<1$, such that

$$
|s-t|>\delta \quad \text { if } \quad s \in G_{i}, t \in G_{j}, \quad i \neq j .
$$

With the given form of $r_{i j}$ and $s_{i j}$, apply (3.19) to the absolute difference in (3.18): the latter is bounded above by

$$
\begin{gathered}
\sum_{\delta u g(u)<j \leqq[u g(u)]}(2 \pi)^{-1} u g(u) r(T / u g(u))\left[1-r^{2}(j T / u g(u))\right]^{-1 / 2} \\
\cdot \exp \left[-u^{2} / 1+|r(j T / u g(u))|\right] .
\end{gathered}
$$

Under (3.2), $r$ is bounded away from 1 outside every neighborhood of 0 ; thus, there exists $b>0$ such that

$$
r(j T / u g(u)) \leqq 1-b, \quad \text { for } j>\delta u g(u) .
$$

It follows that the sum (3.20) is at most equal to

$$
(2 \pi)^{-1} u^{2} g^{2}(u)(1-b) b^{-1 / 2} \exp \left[-u^{2} /(2-b)\right] \text {. }
$$

This function of $u$ is, under the hypothesis on $g$, of smaller order than $\exp \left(-u^{2} / 2\right)$ for $u \rightarrow \infty$; therefore, the expression (3.18) converges to 0 .

We now return to:

Proof of Theorem 3.1. For the given $T>0$ and arbitrary $\varepsilon$, $0<\varepsilon<1$, let the integer $m \geqq 1$ be chosen so large that $T / m$ is smaller than the number $\tau$ in Lemma 3.1 and also so small that $T \gamma(1+\varepsilon) / m<\pi / 2$. Construct the intervals $I_{1}, \cdots, I_{m}$ in the following way. Decompose [0,T) into $m$ subintervals of equal length $T / m$, closed on the left and open on the right. For an arbitrary, small $h>0$, clip the open segment of length $h$ from the right end of each of the $m$ given subintervals; there remain $m$ closed subintervals

$$
I_{j}=[(j-1) T / m, j T / m-h], \quad j=1, \cdots, m .
$$

Let $J_{1}, \cdots, J_{m}$ be the closures of the complementary intervals in $[0, T]$ :

$$
J_{\jmath}=[j T / m-h, j T / m], \quad j=1, \cdots, m ;
$$

each is of length $h$. By the inclusion $I \subset[0, T]$ and Boole's inequality, we have:

$$
\begin{aligned}
& P[\max (X(t): t \in I)>u] \leqq P[\max (X(t): 0 \leqq t \leqq T)>u] \\
& \leqq P[\max (X(t): t \in I)>u]+P[\max (X(t): t \in J>u]
\end{aligned}
$$

Let $K$ and $\sigma$ be the numbers in Lemma 3.2 ; if $h>\sigma$, then, by (3.2) 
and Lemma 3.2, the limiting values of the ratio

$$
\frac{P[\max (X(t): t \in I)>u]}{P[\max (X(t): 0 \leqq t \leqq T)>u]}
$$

fall between $1-K h$ and 1. By Lemma 3.3, the same is true of the ratio

$$
\frac{P[\max (X(t): t \in G)>u]}{P[\max (X(t): 0 \leqq t \leqq T)>u]} .
$$

By Lemma 3.4 and the stationarity of the process, the result extends to the limiting values of the ratio

$$
\frac{1-P^{m}\left(\max \left(X(t): t \in G_{1}\right) \leqq u\right]}{P[\max (X(t): 0 \leqq t \leqq T)>u]} .
$$

By the relation $1-P^{m} \sim m(1-P), P \rightarrow 1$, the ratio above is asymptotic to

$$
\frac{m P\left[\max \left(X(t): t \in G_{1}\right)>u\right]}{P[\max (X(t): 0 \leqq t \leqq T)>u]} .
$$

By another application of Lemma 3.3 (stated for the case $m=1$ ) this is asymptotic to

$$
\frac{m P\left[\max \left(X(t): t \in I_{1}\right)>u\right]}{P[\max (X(t): 0 \leqq t \leqq T)>u]} .
$$

By Lemma 3.1 (with $T / m-h$ in place of $T$ ) this is bounded above by

$$
\frac{m P[\max (Y(t): 0 \leqq t \leqq(T / m-h) \gamma(1+\varepsilon))>u]}{P[\max (X(t): 0 \leqq t \leqq T)>u]}
$$

which, by (3.4), is asymptotic to

$$
\frac{(T-m h) \gamma(1+\varepsilon) \exp \left(-u^{2} / 2\right) / 2 \pi}{P[\max (X(t): 0 \leqq t \leqq T)>u]} .
$$

From the statement about the limiting values of the ratio (3.22) and the relations following it, we infer :

$$
\liminf _{u \rightarrow \infty} \frac{(T-m h) \gamma(1+\varepsilon) \exp \left(-u^{2} / 2\right) / 2 \pi}{P[\max (X(t): 0 \leqq t \leqq T)>u]} \geqq 1-K h
$$

By similar reasoning, with $1-\varepsilon$ in place of $1+\varepsilon$, we obtain :

$$
\limsup _{u \rightarrow \infty} \frac{(T-m h) \gamma(1-\varepsilon) \exp \left(-u^{2} / 2\right) / 2 \pi}{P[\max (X(t): 0 \leqq t \leqq T)>u]} \leqq 1 .
$$

Let $h \rightarrow 0$; then the left hand members depend only on $\varepsilon$ but not $m$. 
Since $\varepsilon>0$ is arbitrary, the relation (3.3) follows, and the proof of the theorem is complete.

4. Invariance of the limiting distribution of $L$ under changes in T. Now we write $L$ as $L(T)$ to display its dependence on $T$.

THEOREM 4.1. For every $T>0$ and every integer $m \geqq 1$, we have :

$$
\frac{P[0<L(T) \leqq x / u]}{P[L(T)>0]} \sim \frac{P[0<L(T / m) \leqq x / u]}{P[L(T / m)>0]}, \quad u \rightarrow \infty,
$$

for all $x>0$.

Proof. For $m \geqq 1$, we have:

$$
\lim _{u \rightarrow \infty} \frac{P[X(j T / m)>u, \text { for some } j=0,1, \cdots, m]}{P[L(T)>0]}=0 ;
$$

indeed, by Theorem 3.1, the denominator is of the order $\exp \left(-u^{2} / 2\right)$, and, by Boole's inequality, the numerator is at most

$$
m P[X(0)>u]=\exp \left(-u^{2} / 2\right) \cdot 0\left(u^{-1}\right) .
$$

Let $N$ be the number of upcrossings of $u$ by $X(t), 0 \leqq t \leqq T$; then, (4.1) implies:

$$
P[N \geqq 1] \sim P[L(T)>0],
$$$$
u \rightarrow \infty \text {. }
$$

For, on the one hand, if both $N=0$ and $L(T)>0$, then $X(0)$ must exceed $u$; thus :

$$
P[L(T)>0]=P[N \geqq 1, L(T)>0]+0(1-\Phi(u)), \text { for } u \rightarrow \infty,
$$

so that (4.1) implies:

$$
P[L(T)>0] \sim P[N \geqq 1, L(T)>0] .
$$

On the other hand, the latter implies (4.2) because $N \geqq 1$ only if $L(T)>0$.

Next we obtain :

$$
\lim _{u \rightarrow \infty} \frac{P[N \geqq 2]}{P[N \geqq 1]}=0 ;
$$

in fact, 


$$
\begin{aligned}
\frac{P[N \geqq 2]}{P[N \geqq 1]} & =\frac{P[N=1]+2 \sum_{k=2}^{\infty} P[N=k]}{\sum_{k=1}^{\infty} P[N=k]}-1 \leqq \frac{\sum_{k=1}^{\infty} k P[N=k]}{\sum_{k=1}^{\infty} P[N=k]}-1 \\
& =\frac{E N}{P[N \geqq 1]}-1,
\end{aligned}
$$

and the last expression tends to 0 because $E N$ is equal to the right hand side of (3.3), and $P[N \geqq 1]$ is, by (4.2), asymptotic to the right hand side of (3.3).

If $L(T)>0$ and $X(j T / m) \leqq u$ for $j=0,1, \cdots, m$, then $N \geqq 1$; therefore, from (4.1), (4.2) and (4.3), we get:

$$
\begin{aligned}
& \frac{P[0<L(\mathrm{~T}) \leqq x / u]}{P[L(T)>0]} \sim \\
& \frac{P[0<L(T) \leqq x / u ; X(j T / m) \leqq u, 0<j \leqq m ; N=1]}{P[L(T)>0]}, u \rightarrow \infty,
\end{aligned}
$$

The event in the numerator on the right hand side of (4.4) is that an excursion above $u$ takes place within exactly one of the open subintervals of length $\mathrm{T} / m$ and that the duration is not more than $x / u$. If the excursion takes place in $((i-1) T / m, i T / m)$ then

$$
L(T)=L(i T / m)-L((i-1) T / m) ;
$$

therefore ;

$$
\begin{aligned}
& P[0<L(T) \leqq x / u ; X(j T / m) \leqq u, 0 \leqq j \leqq m ; N=1] \\
& \begin{aligned}
=\sum_{i=1}^{m} P[0<L(i T / m)-((i-1) T / m) & \leqq x / u ; X(j T / m) \\
& \leqq u, 0 \leqq j \leqq m ; N=1] .
\end{aligned}
\end{aligned}
$$

By the same argument supporting (4.4) we have:

$\underline{P[0<L(i T / m)-L((i-1) T / m) \leqq x / u ; X(j T / m) \leqq u, 0 \leqq j \leqq m ; N=1]}$

$$
P[L(T)>0]
$$

$$
\begin{aligned}
& \sim \frac{P[0<L(i T / m)-L((i-1) T / m) \leqq x / u]}{P[L(T)>0]} \\
& =\frac{P[0<L(T / m) \leqq x / u]}{P[L(T)>0]}(\text { by stationarity }), \quad i=1, \cdots, m .
\end{aligned}
$$

From (4.4), (4.5) and (4.6), it follows that

$$
\frac{P[0<L(T) \leqq x / u]}{P[L(T)>0]} \sim \frac{m P[0<L(T / m) \leqq x / u]}{P[L(T)>0]} .
$$


It is clear from (3.3) that

$$
P[L(T)>0] \sim m P[L(T / m)>0] ;
$$

therefore, (4.7) implies the assertion of the theorem.

5. The main result and its extensions.

THEOREM 5.1. Let $X(t), t \geqq 0$, be a stationary Gaussian process satisfying the conditions stated at the beginning of $\S 3$ : then

$$
\lim _{u \rightarrow \infty} \frac{P[0<\gamma L(T) \leqq x / u]}{P[L(T)>0]}=\Psi(x / 2)
$$

for every positive $x$ and $T$.

Proof. By the moment convergence theorem (cf. $\S 1$ ) it suffices to show that the moments of the conditional distribution converge to the corresponding moments of the limit distribution.

Let $\varepsilon, 0<\varepsilon<1$, be arbitrary. By Theorem 4.1, we may assume that $T$ is as small as we want; thus, it will be supposed to be smaller than $\tau$, where $\tau$ is the number for which (3.6) holds. For the process $Y(t)$ in $(1.1)$, let $L(T,+)$ be the time spent above $u$ by $Y((1 \pm \varepsilon) \gamma t), 0 \leqq t \leqq T$. Lemma 2.1 and the inequalities (3.6) imply:

$$
\begin{array}{rlr}
E\left[L^{n}(T,+) \mid L(T,+)>0\right] \leqq E\left[L^{n}(T) \mid L(T)>0\right] & \\
& \leqq E\left[L^{n}(T,-) \mid L(T,-)>0\right], & n \geqq 1 .
\end{array}
$$

By Lemma 1.3, the extreme members of this inequality converge to the moments of the limiting distribution; therefore, the middle member also does. The normalizing constant $\gamma$ cancels the factor $1 / \gamma$ arising from the transformation of the excursion integral of $Y(\gamma t)$ to $Y(t)$.

REMARKs. The essential condition on the process is (3.1). It follows from the well known criterion for Gaussian processes [1] that (3.1) implies that there exists a version of the process with continous sample functions. Theorem 5.1 is valid for this version without a special continuity assumption.

The roots of the equation $1-r(t)=0$ form a closed subgroup of the reals: either (3.2) holds or there exists $\lambda>0$ such that $r(\lambda n)=1$ for all integers $n$ and $r(t)<1$ if $t \neq \lambda n$ for some $n$. In the latter case $X(t)$ is periodic with period $\lambda$, and the process is of interest only on the parameter set $0 \leqq t \leqq \lambda$. All of our results are valid for $T \leqq \lambda$. 


\section{REFERENCES}

1. Ju. K. Beljaev, Continuity and Hölder's conditions for sample functions of stationary Gaussian processes, Proc. Fourth Berkeley Sympos. Math. and Prob., 2 Contributions to probability Theory, Univ. of California Press, Berkeley, Calif. 1961, 23-33.

2. Ju. K. Beljaev and V. P. Nosko, Characteristics of excursions over a high level for a Gaussian process and its envelope, Teoria Verojatnos i. Primenen. 14 (1969), 302314.

3. S. M. Berman, Limit theorems for the maximum term in stationary sequences, Ann. Math. Stat. 35 (1964), 502-516.

4. H. Cramér and M. R. Leadbetter, Stationary and Related Stochastic Processes: Sample Function Properties and their Applications, John Wiley, New York, 1967.

5. M. Kac and D. Slepian, Large excursions of Gaussian processes, Ann. Math. Stat. 30 (1959), 1215-1228.

6. M. Loeve, Probability Theory, Third edition, Van Nostrand, Princeton, 1963.

7. D. S. Palmer, Properties of random functions, Proc. Camb. Phil. Soc. 52 (1956), $672-686$.

8. S. O. Rice, Duration of fades in radio transmission, Bell System Tech. J. 37 (1958), 581-635.

9. D. Slepian, The one-sided barrier problem for Gaussian noise, Bell System Tech. J. 41 (1962), 463-501.

10. V. A. Volkonskii and Ju. A. Rozanov, Some limit theorems for random functions II, Theory Prob. Applic. 6 (1961), 186-198 (English translation).

Reference added in proof: J. Lamperti, On limit theorems for Gaussian processes, Ann. Math. Stat. 36 (1965), 304-310.

Received February 3, 1970. This paper represents results obtained at the Courant Institute of Mathematical Sciences, New York University, under the sponsorship of the National Science Foundation Grant NSF-GP-11460.

NEW YORK UNIVERSITY 



\title{
PACIFIC JOURNAL OF MATHEMATICS
}

\author{
EDITORS
}

H. SAMELSON

Stanford University

Stanford, California 94305

C. R. Новву

University of Washington

Seattle, Washington 98105
J. DUGUNDJI

Department of Mathematics

University of Southern California

Los Angeles, California 90007

RICHARD ARENS

University of California

Los Angeles, California 90024

\section{ASSOCIATE EDITORS}

E. F. BECKENBACH

B. H. NeUManN

F. WOLF

K. YosHidA

\section{SUPPORTING INSTITUTIONS}

UNIVERSITY OF BRITISH COLUMBIA CALIFORNIA INSTITUTE OF TECHNOLOGY UNIVERSITY OF CALIFORNIA MONTANA STATE UNIVERSITY UNIVERSITY OF NEVADA NEW MEXICO STATE UNIVERSITY OREGON STATE UNIVERSITY UNIVERSITY OF OREGON OSAKA UNIVERSITY UNIVERSITY OF SOUTHERN CALIFORNIA
STANFORD UNIVERSITY UNIVERSITY OF TOKYO UNIVERSITY OF UTAH WASHINGTON STATE UNIVERSITY UNIVERSITY OF WASHINGTON

AMERICAN MATHEMATICAL SOCIETY CHEVRON RESEARCH CORPORATION TRW SYSTEMS

NAVAL WEAPONS CENTER 


\section{Pacific Journal of Mathematics}

\section{Vol. 36, No. $1 \quad$ November, 1971}

Norman Larrabee Alling, Analytic and harmonic obstruction on

nonorientable Klein surfaces ............................ 1

Shimshon A. Amitsur, Embeddings in matrix rings .............. 21

William Louis Armacost, The Frobenius reciprocity theorem and essentially bounded induced representations ....................... 31

Kenneth Paul Baclawski and Kenneth Kapp, Topisms and induced

non-associative systems ............................ 45

George M. Bergman, The index of a group in a semigroup ............ 55

Simeon M. Berman, Excursions above high levels for stationary Gaussian

processes....................................... 63

Peter Southcott Bullen, A criterion for $n$-convexity .............. 81

W. Homer Carlisle, III, Residual finiteness of finitely generated commutative

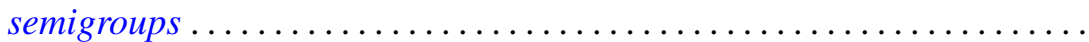

Roger Clement Crocker, On the sum of a prime and of two powers of

two ............................................ 103

David Eisenbud and Phillip Alan Griffith, The structure of serial rings . . . 109

Timothy V. Fossum, Characters and orthogonality in Frobenius

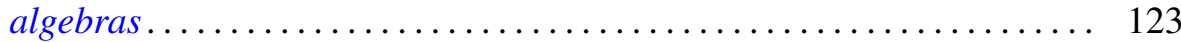

Hugh Gordon, Rings of functions determined by zero-sets . .......... 133

William Ray Hare, Jr. and John Willis Kenelly, Characterizations of Radon partitions...

Philip Hartman, On third order, nonlinear, singular boundary value

problems

David Michael Henry, Conditions for countable bases in spaces of

countable and point-countable type ..

James R. Holub, Hilbertian operators and reflexive tensor products ...

Robert P. Kaufman, Lacunary series and probability ..... . .

195

Erwin Kreyszig, On Bergman operators for partial differential equations in

two variables ................................

Chin-pi Lu, Local rings with noetherian filtrations . .

Louis Edward Narens, A nonstandard proof of the Jordan curve theorem...

S. P. Philipp, Victor Lenard Shapiro and William Hall Sills, The Abel summability of conjugate multiple Fourier-Stieltjes integrals. .

Joseph Earl Valentine and Stanley G. Wayment, Wilson angles in linear normed spaces

Hoyt D. Warner, Finite primes in simple algebras ...

Horst Günter Zimmer, An elementary proof of the Riemann hypothesis for an elliptic curve over a finite field... 